\title{
Improving Pre-hospital Care of Road Traffic Accident's Victims with Smartphone Technology
}

\author{
https://doi.org/10.3991/ijim.v12i2.8118 \\ Osman A. Abdellah, Majed M. Aborokbah, Abdelrahman Osman Elfaki $\left.{ }^{\bowtie}\right)$ \\ University of Tabuk, Tabuk, Saudi Arabia \\ a.elfaki@ut.edu.sa
}

\begin{abstract}
One of the most causes to lose millions of lives around the world is Road Traffic Accidents (RTAs). According to the world health organization (WHO) report, 1.25 million people are killed each year as a result of RTAs, 20 to 50 million people were injured, and the number of killed people by RTAs is expected to increase further by 2020 . The recent studies conclude that patient survival during a health emergency situation depends on the effective prehospital healthcare services, while the effective communication between the paramedics and prehospital staff is one of the important healthcare success factors. With the rapid growing of information and communication technology (ICT), wireless technologies and mobile services can provide viable solution to overcome the pre-hospital healthcare problems. The aim of this research is to improve the quality of prehospital emergency healthcare services at KSA by developing and implementing a mobile based emergency system. The proposed application is moving the diagnosis time to be started during traveling time witch accelerate the treatment. The proposed system shows satisfactory results in term of effectiveness and satisfaction.
\end{abstract}

Keywords—-prehosptal, mobile based application, road traffic accident's victims care

\section{Background and Motivation}

One of the most causes to lose millions of lives around the world is Road Traffic Accidents (RTAs). According to a report released by World Health Organization (WHO) 2013, 1.25 million people are killed each year as a result of RTAs, 20 to 50 million people were injured, and the number of killed people by RTAs is expected to increase further by 2020 [1,2]. Unfortunately, Saudi Arabia (KSA) is no exception to this tragic trend; The KSA has been categorized as one of the country with highest rate of RTAs in the region [3].

Recent statistics indicate that RTCs is prevalent world-wide. Table 1 illustrates RTCs prevalence among some countries. 
Table 1. 26 Countries with highest road traffic deaths prevalence

\begin{tabular}{|c|l|c|c|l|c|}
\hline Rank & Country & $\begin{array}{c}\text { Estimated } \\
\text { road traffic } \\
\text { death rate } \\
\text { per 100 000 } \\
\text { population** }\end{array}$ & Rank & Country & $\begin{array}{c}\text { Estimated road } \\
\text { traffic death } \\
\text { rate per 100 } \\
\text { 000 popula- } \\
\text { tion* }\end{array}$ \\
\hline 1 & Libya & 73.4 & 14 & Gambia & 29.4 \\
\hline 2 & Thailand & 36.2 & 15 & Dominican Republic & 29.3 \\
\hline 3 & Malawi & 35 & 16 & Kenya & 29.1 \\
\hline 4 & Liberia & 33.7 & 17 & Madagascar & 28.4 \\
\hline 5 & Democratic Republic of the Congo & 33.2 & 18 & Zimbabwe & 28.2 \\
\hline 6 & United Republic of Tanzania & 32.9 & 19 & Benin & 27.7 \\
\hline 7 & Central African Republic & 32.4 & 20 & Cameroon & 27.6 \\
\hline 8 & Rwanda & 32.1 & 21 & Guinea-Bissau & 27.5 \\
\hline 9 & Iran & 32.1 & 22 & Saudi Arabia & 27.4 \\
\hline 10 & Mozambique & 31.6 & 23 & Uganda & 27.4 \\
\hline 11 & Togo & 31.1 & 24 & Guinea & 27.3 \\
\hline 12 & Sao Tome and Principe & 31.1 & 25 & Sierra Leone & 27.3 \\
\hline 13 & Burkina Faso & 30 & 26 & Senegal & 27.2 \\
\hline
\end{tabular}

In KSA, RTCs are a national problem adversely impacting negatively in economy and societal fabric [2; 4], from an economic perspective the RTCs are most costly, this cost is spent on victim's treatment, and those killed or permanently disabled may cause reduced productivity.

The mHealth is an abbreviation for mobile health technology; a term is used to refer to the integration of mobile devices, computing, medical sensors, and portable devices to ensure healthcare [5]. In recent years the mhealth has been growing exponentially. About 808 mHealth articles have been indexed in PubMed between 2013 and January 2015. Likewise, indexed mHealth articles in Scopus have seen an exponential increased since $2010[6,7]$. Consequently the numbers of publication are likely to increase further.

The recent studies conclude that patient survival during a health emergency situation depends on the effective pre-hospital healthcare services. As is necessarily known, a successful treatment is based on accurate and fast diagnosis. Fast diagnosis of a casualty helps greatly in his survival. Diagnosis of a casualty shall only begin after his arrival at the hospital as the mission of the paramedics is to preserve his life. In this paper, a mobile application system has been presented to accelerate the diagnosis process, as it will start at the Ambulance.

The proposed application, a mobile based prehospital emergency system, provide a direct communication between emergency doctors and paramedics which allow them to exchange text, images, and videos before reaching hospital. This information will assist doctors to early evaluate the patient situation and start diagnosis before reaching hospital. In addition, paramedics could receive the doctors' directions that help to save patient life in case needed. 
In the sections that follow, we present related works; this is followed by a detailed discussion on the research methodology, presents the achieved results, and concludes the summarizing of objectives and findings.

\section{Related works}

Currently there are several studies done in mobile based prehospital emergency healthcare area, in this section a brief summary of the recent related works are presented below.

An electronic record system with use of mobile devices (tablets) focuses on prehospital patient care have been developed and implemented by [8], the application aims to improve the delivered prehospital care service through enabling the collection of patient data, this data will synchronized with a remote server and stored in a database for analysis and studies purposes.

[9] propose a mobile emergency care management system using WebRTC technology for real time communication. The system is easy to deploy and requires internet connection and chrome or Mozilla browser to work properly. The initial assessment of the patient is carried within the ambulance using the mobile device patient and later the patient is brought to the nearest health center aims to provide an extra treatment by the assistance of specialists whose telepresence are provided by mobile with WebRTC technology.

A comprehensive mobile e-healthcare system has been provided by [10] in order to provide good patient monitoring system in indoor and outdoor. The system architecture is contains two main parts, the first part is mobile phone application in a smart phone and linked with Bio-sensors, the second part is a database center linked with several healthcare centers and ambulance centers. The mobile application is developed to implement the main following functions: decide the patient status based on the recorded Bio-signals from sensors, send important information to the patient record, then allow physicians to access to any patient record.

[11] propose a mobile based Pre-hospital Emergency Notification System to allow the emergency medical staff to inform the hospital team about the incoming victim's personal information and severity level of road accident victims. Furthermore, the system allow the hospital staff to view the incoming victims information by provides a web-based technology. The mobile application has been developed using App Inventor 2, PHP and MYSQL.

A smart real-time prehospital communication system by using mobile device, wearable sensing and video technology has been developed to enhance the emergency medical service (EMS), to support multi-dimensional telemetry monitoring a costeffective wearable physiological sensing solution is introduced, and then a real-time data sharing platform based on cloud services is constructed in order to allow the sending of all collected prehospital data to the hospital before to ambulance arrival aiming to give a more complete information about the incoming patient situation [12].

[13] present an emergency healthcare system using mobile device and cloud services, in this system the cloud-based architecture has been developed to enable au- 
thorized users to access emergency case information, and to select the most suitable treatment procedures for the case and select the most suitable ambulances and hospitals for each situation. Further the cloud-based services allow the sharing and exchange of information between EMS agencies and hospitals.

The rescuer supporting system for the prehospital emergency medical service (ARSA) has been proposed by [14]. The system aims to help the patients through allow the service of sending an emergency request to the nearest paramedics and the medical agencies transporters. This system the emergency service should be provided directly to the patient without waiting or consuming time for the ambulance arrival from the EMS agency. This might increases the survival rate of the emergency patients.

Furthermore, in [1] a prehospital health care management platform has been presented; this platform aims to support the emergency health care provided services from the beginning of an emergency call until a patient is arrived to hospital center, the platform divided into the following three main parts: emergency supports dispatch protocol system, ambulance data recording and workflow management, and emergency telemedicine system.

\section{The Proposed System}

The main idea of the proposed pre-hospital emergency system is to accelerate the diagnosis time for a casualty by providing bidirectional communication tool between emergency doctors and paramedics, the proposed application has follow the concepts of telemedicine. The proposed application will provide patient's information in different standard format such as images, text, voice, and video.

The total time from arrival of the ambulance to the injured to the beginning of treatment could be defined as traveling time plus diagnosis time. As a fact, treatment could not be started until finishing the diagnosis and diagnosis is starting after finishing traveling time. In the following, mathematical representation had been discussed in sake of explain the contribution.

Definition 1: Let Tt denotes treatment time, Dt denotes diagnosis time, and TVt denotes traveling time, then:

$\mathrm{Tt}=\mathrm{f}(\mathrm{Dt}, \mathrm{TVt})$. In considering $\mathrm{Tt}$, Dt is a major factor and TVt a supporting factor, which means the impact of $\mathrm{Dt}$ is higher than the impact of TVt. hence the real translation should be $\mathrm{Tt}=\mathrm{f}(\mathrm{Dt}, \mathrm{K}(\mathrm{TVt}))$. Where $\mathrm{K}<1$.

The contribution of the proposed application is to reduce diagnostic time by transferring part of it to traveling time. This reducing is achieved by providing a supportive communication tool (through the application) to link the ambulance paramedics and Emergency Physicians. In RTA, The diagnostic process depends largely on the results of the radiation therapy. Hence, diagnosis time could be defined to three times: first diagnosis time where a suitable diagnostic radiology (X ray, CT scan, etc) should be selected, second diagnosis time where the selected diagnostic radiology is running and third diagnosis time where the results of diagnostic radiology is extracted and explained. In some case, less than 10 percent, where is no need for a diagnostic 
radiology When there is no need for diagnostic radiation, the diagnostic time is a time of patient examination $(\mathrm{Dt}=\mathrm{EDt})$. In this paper, we have focused only on the first situation where there is severe need for diagnostic radiation.

Definition 2: Dt $=D 1 t+D 2 t+D 3 t$. Where:

Dt is a diagnosis time.

D1t is a selection time for suitable diagnostic radiology.

D2t is a time of diagnostic radiology.

D3t is a results time.

EDt is a patient examination time.

By substitution of definition 2 in definition 1 the treatment is a function of:

$\mathrm{Tt}=\mathrm{f}((\mathrm{D} 1 \mathrm{t}+\mathrm{D} 2 \mathrm{t}+\mathrm{D} 3 \mathrm{t}), \mathrm{TVt})$. The proposed application allows emergency doctors to selected the suitable diagnostic radiology within traveling time, hence the new traveling time $\mathrm{TVt}=\mathrm{TVt}+\mathrm{D} 1 \mathrm{t}$. And treatment time will be function of $\mathrm{Tt}=\mathrm{f}((\mathrm{D} 2 \mathrm{t}+$ D3t), TVt).

The proposed application has been developed by using prototype methodology. Three versions had been developed until the final version has been approved by our stakeholders. Table 2 shows the descriptions of the development process.

Table 2. Descriptions of the development process

\begin{tabular}{|c|l|l|}
\hline Version & \multicolumn{1}{|c|}{ Features } & \multicolumn{1}{c|}{ Stakeholders comments } \\
\hline 1 & Sending text, pictures & Not enough for diagnosis process \\
\hline 2 & Sending text, pictures, or audio. & Not enough for diagnosis process \\
\hline 3 & Sending text, pictures, audio, or video & Not enough for diagnosis process \\
\hline 4 & $\begin{array}{l}\text { Sending text, pictures, audio, or video and } \\
\text { allow bidirectional communication }\end{array}$ & Approved \\
\hline
\end{tabular}

The proposed system architecture is divided into three main parts as shown in Figure 1: mobile or tablet device with pre-hospital emergency application to be present within the ambulance, web server device at hospital side that operates by hospital staff to receive information from ambulance, and health professional on computer or laptop with Internet to connect to the server. Figure 1 explains the architecture of the proposed system.

The proposed pre-hospital emergency application is developed for the hand-held smart device Samsung phones with Android 7 or later. The device requires the following: a built-in camera, microphone and Wi-Fi which the application needs for communication between ambulance and hospital staff.

Figure 2 shows the interactions and main processes in the proposed application. 


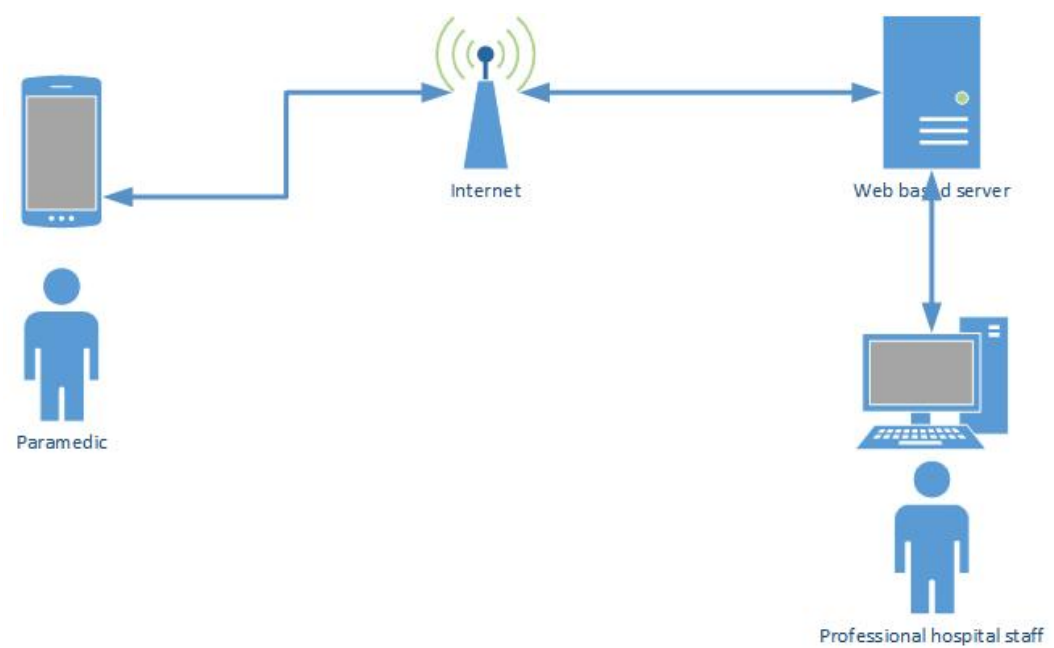

Fig. 1. The proposed system architecture

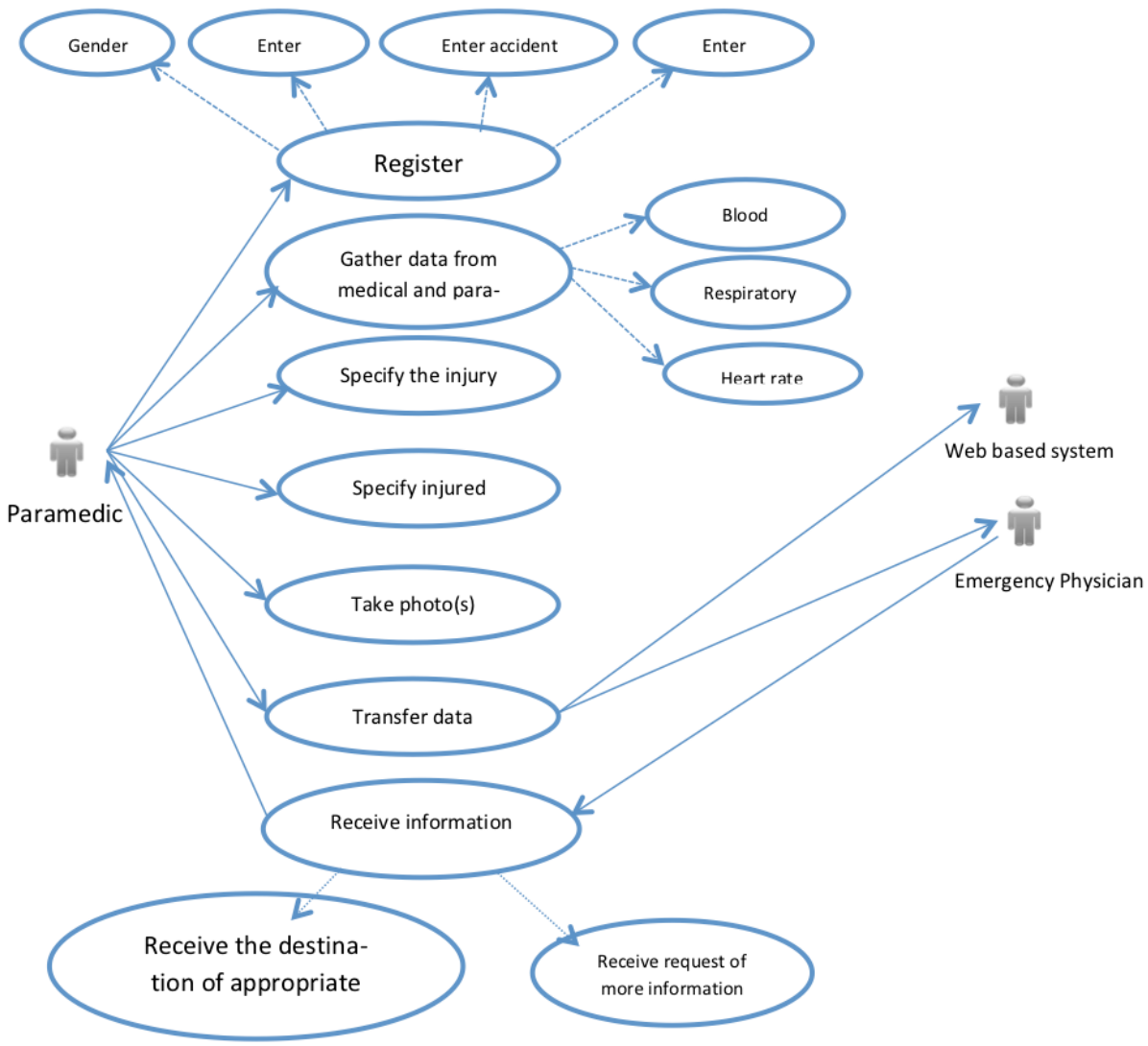

Fig. 2. The interactions and main processes in the proposed application. 


\section{$4 \quad$ Results}

The realized application begins with a first user interface, intended to recognize the user and to propose the various application functionalities. Refer to Figure 3 for the splash, login, and main screens of the mPrehospital application.

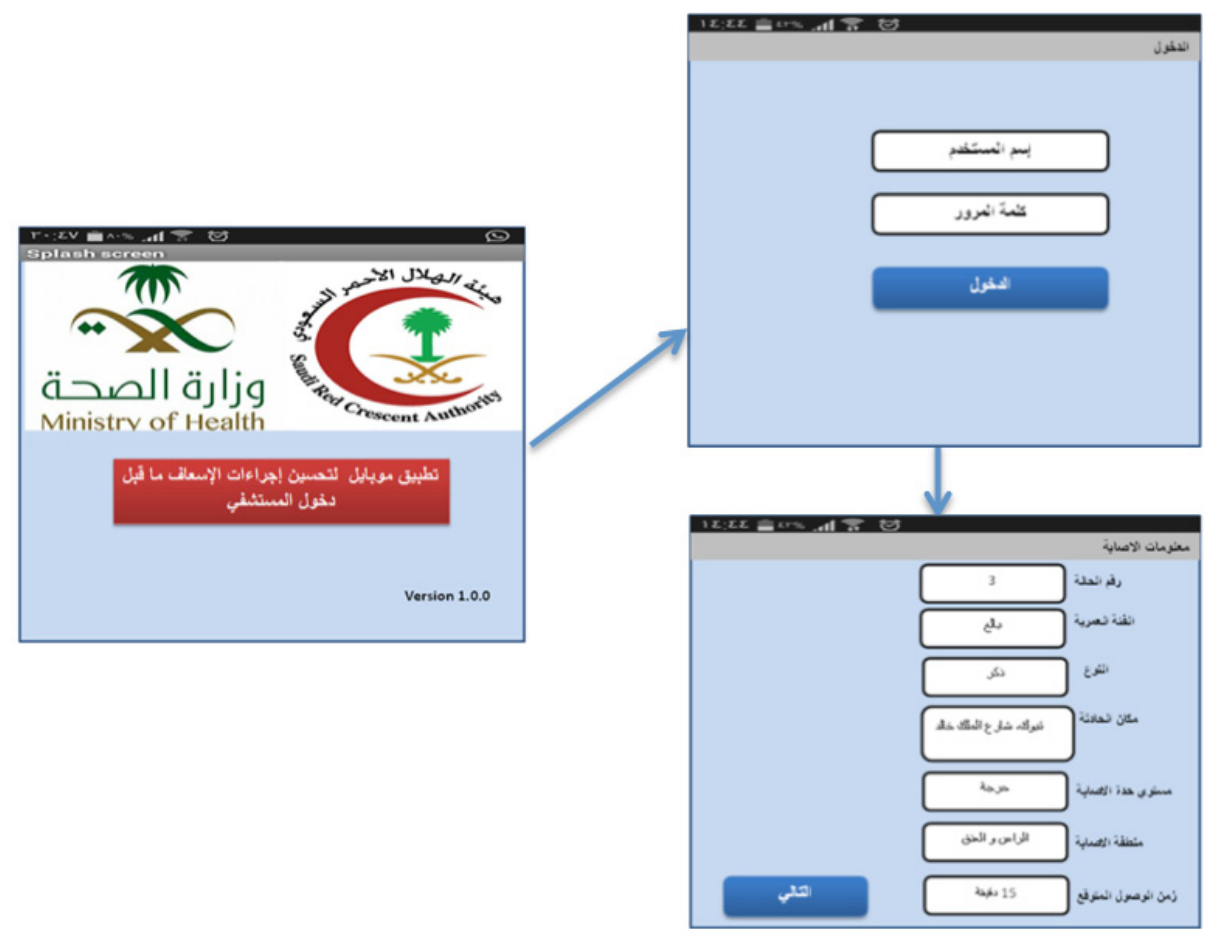

Fig. 3. The opening of splash screen (1) allows the paramedic to start working in mPrehospital app., then the login screen (2) will appear after 15 seconds which allows the paramedic to enter their user name and password, Once the "login" button is clicked, the main screen (3) opens up to fill the injured information.

A web based system is developed to receive the information of incoming victims from paramedics as shown in Figure 4. 


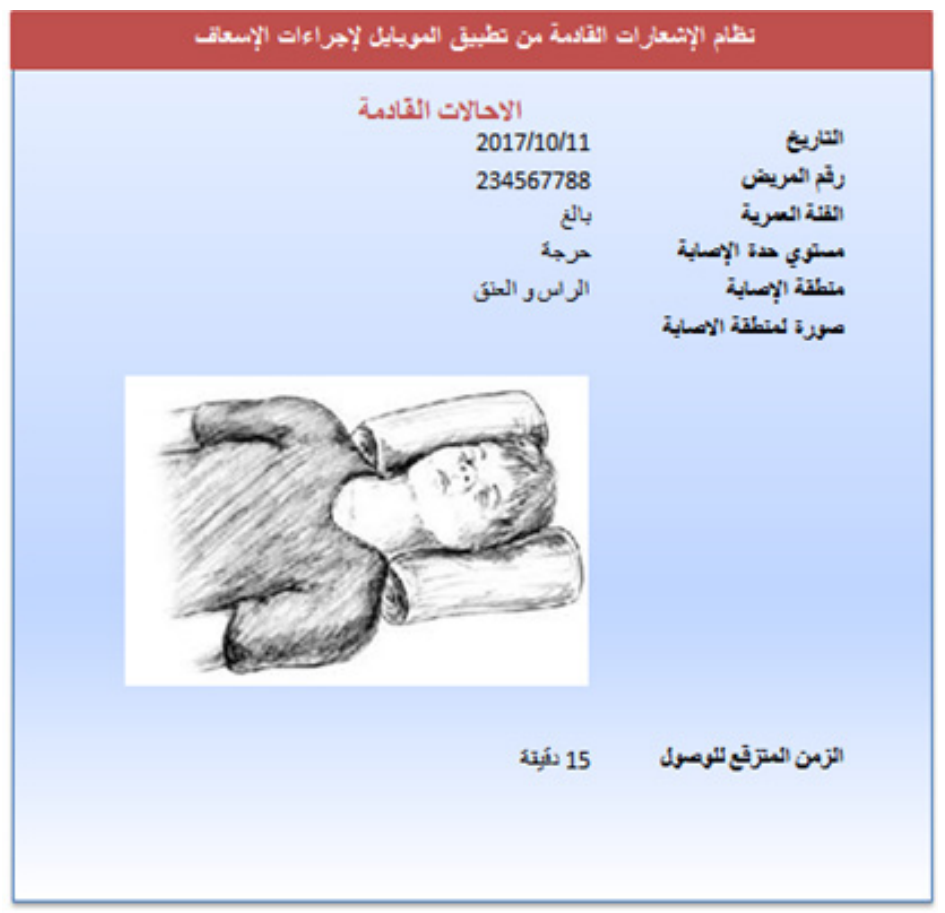

Fig. 4. Web based prehospital system

\subsection{Usability evaluation}

Upon the completion of implementation phase, the usability evaluation process of mobile applications was carried out based on ISO/IEC 9126-4 Metrics recommends [16] and with the following metrics:

Effectiveness: The completion rate is used to measure the completeness of all tasks given to users; due its simplicity and ease of understand the completion rate is most popular effectiveness metric. Effectiveness can thus be represented as a percentage by using the following simple equation:

Effectivenesses $=\frac{\text { Number of task completed successfully }}{\text { Total number of task undertaken }} \times 100 \%$

Twenty-three (23) participants from two main application users (11 ambulance users and 12 hospital users) are participated to complete all tasks given (Task 1 to Task 5). Figure 5 shows the results of completion rate based on the users responses. 


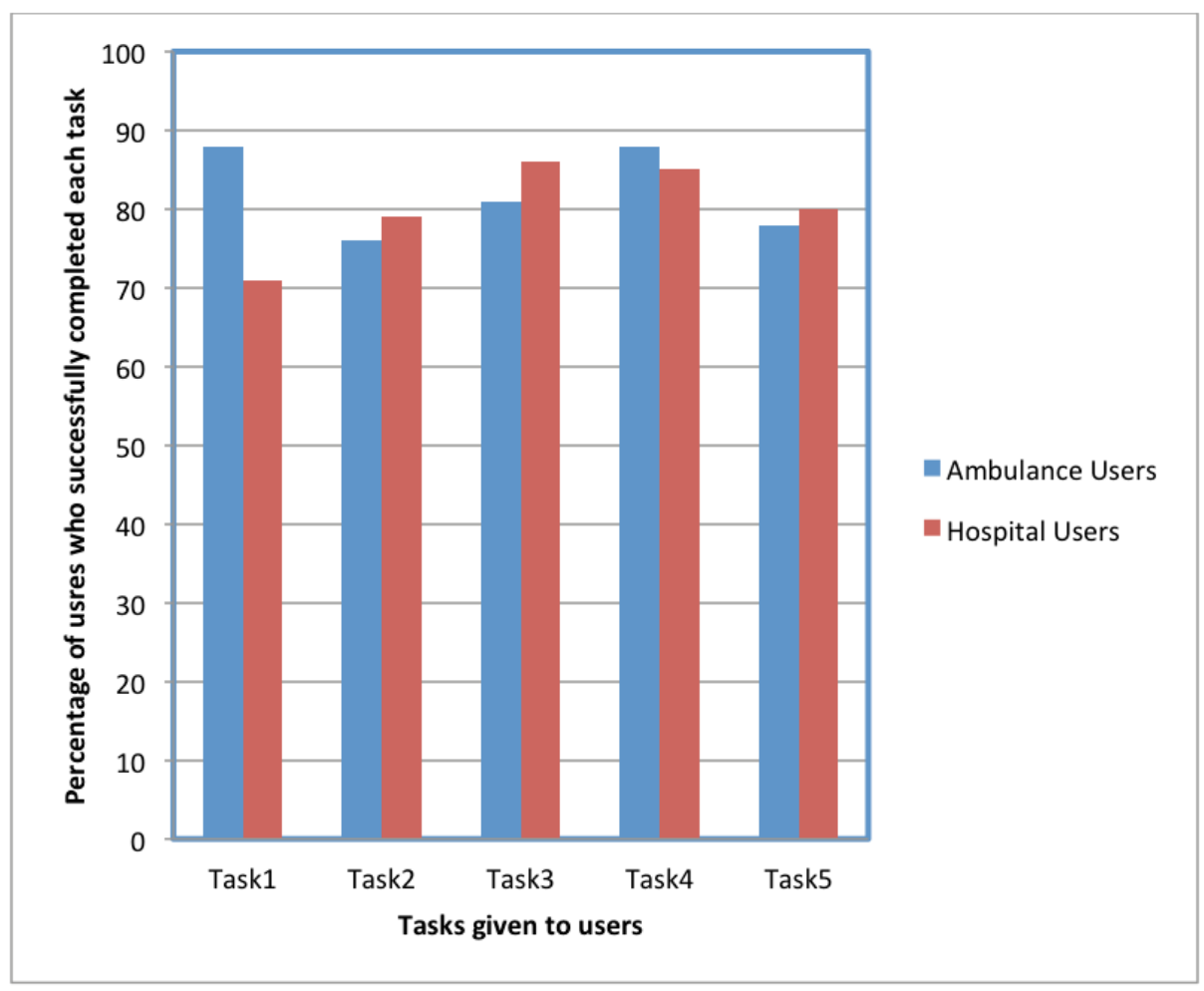

Fig. 5. Completion rate results

Satisfaction: The after scenario questionnaire (ASQ) technique was developed by [17], its standardized satisfaction questionnaires which can be conducted upon completion each task and/or upon completion of the usability test session. Normally, the user can circle their answer using the provided 7 point scale, where 1 denotes strongly agree and 7 denotes strongly disagree. After the user has completed the ASQ, the ASQ score can be calculated by taking the average (with the arithmetic mean) of the 3 questions. In case of participant skipped the answer of question or marked N/A skipped, the ASQ can be calculated by averaging the remaining scores.

Figure 6 shows the user satisfaction. The evaluation had been conducted to measure the satisfaction of participants through ASQ in term of overall reaction, usability and user interface, application capabilities, and technical manual and help. 


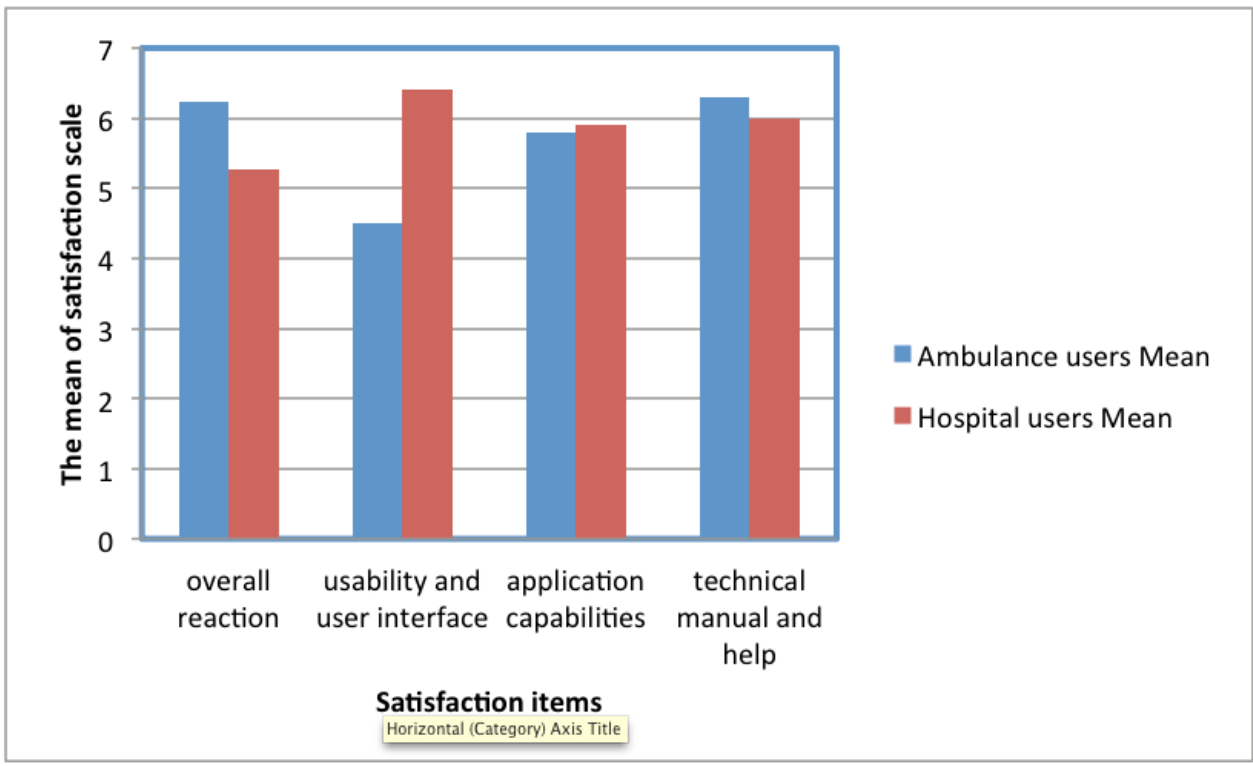

Fig. 6. Satisfaction results

\section{Conclusions}

In this work, mobile application to improve prehospital care of RTAs victims in KSA was developed. The main contribution of this work is to provide effective link between ambulance and hospital staff to accelerate and improve the prehospital service care during emergency situation. Evaluation of the application demonstrates that the realized application is understandable, useful, and effective to provide prehospital care.

The contributions of this research can be summarized as follows:

a) Improving the care services during ambulance movement: This contribution has been attained through providing a supportive way to link the ambulance paramedics and Emergency Physicians. In most RTAs victim's cases the diagnosis time is more critical issues that play a significant role in victims survive. Indeed delays in diagnosis which is the one of most important care dimension for those involved in a RTA increase the severity of injuries. In addition the application provide continuous communication link with Emergency Physicians during the ambulance movement, this might contribute in improving the provided care.

b) Conveying the RTAs victim to appropriate Emergency department: During the care processes in accident location the application provides a direct link between ambulance paramedics and Emergency Physicians then Emergency Physicians can decide the appropriate Emergency department to receive the victim. The Conveying victims to appropriate Emergency department result in reducing the probability of complication occurs and ensuring that they receive appropriate care. 
c) Linking the received victim's information with existing patient care record archive: The linking of received victim information with its patient record, this provides significant information about his record archive which might include: antibiotics resistance, other diseases (i.e. diabetes, asthma, cardiovascular disease). The knowing information about victim medical record archive should help ambulance paramedics treat the victims properly.

\section{Acknowledgment}

This research is funded by the University of Tabuk, Scientific Research Deanship, and project number $0224-1438$.

\section{$7 \quad$ References}

[1] World Health Organization, Road safety in the eastern Mediterranean Region, facts from the global status report on road safety 2013, Available at: (2013) http://www.who.int/vio lenceinjuryprevention/roadsafetystatus/2013 [accessed on 8.06.15]

[2] 6World Health Organization. Global Status Report on Road Safety 2013. Geneva (CH): World Health Organization; 2013.y Available from: http://www.who.int/violence injury prevention/road safety status/2013/en.

[3] World Health Organization, Road traffic injuries, Fact sheet, Reviewed November 2016, Available at: http://www.who.int/mediacentre/factsheets/fs358/en/

[4] Nedal T. Ratrout, Shakhawat Chowdhury, Uneb Gazder, Syed Masiur Rahman, Characterization of crash-prone drivers in Saudi Arabia - A multivariate analysis, Case Studies on Transport Policy, 2016.

[5] R. Istepanian, E. Jovanov, Y. Zhang, Guest editorial introduction to the special section on m-health: beyond seamless mobility and global wireless health-care connectivity, IEEE Trans Inf Technol Biomed, 8 (2004), pp. 405-414 https://doi.org/10.1109/TITB. 2004.840019

[6] J.D. Cameron, A. Ramaprasad, T. Syn, An ontology of mHealth, Proceedings of the 21st Americas Conference on Information Systems (AMCIS 2015), Puerto Rico, USA (2015)

[7] Joshua D. Cameron, Arkalgud Ramaprasad, Thant Syn, An ontology of and roadmap for mHealth research, International Journal of Medical Informatics, Volume 100, April 2017, Pages 16-25.

[8] A. F. Duarte, H. V. Cesar, A. L. M. Marques, P. M. d. A. Marques, and ' G. A. P. Junior, "Prehospital electronic record with use of mobile devices ' in the samu's ambulances in ribeirao preto-brazil," in 2015 IEEE 28th International Symposium on Computer-Based Medical Systems, June 2015, pp. 362-363. https://doi.org/10.1109/CBMS.2015.21

[9] Vidul, A.P., Hari, S., Pranave, K.P., Vysakh, K.J., Archana, K.R.: Telemedicine for emergency care management using WebRTC. In: 2015 International Conference on Advances in Computing, Communications and Informatics (ICACCI), pp. 1741-1745. IEEE (2015) https://doi.org/10.1109/ICACCI.2015.7275865

[10] Majeed Q, Hbail H, Chalechale A (2015) A comprehensive mobile e-healthcare system. In: 2015 7th conference on information and knowledge technology (IKT), IEEE, pp 14Google Scholar. https://doi.org/10.1109/IKT.2015.7288802 
[11] A. Sarlan, F. K. Xiong, R. Ahmad, W. F. W. Ahmad and E. Bhattacharyya, "Pre-hospital emergency notification system," 2015 International Symposium on Mathematical Sciences and Computing Research (iSMSC), Ipon, 2015, pp. 168-173.

[12] X. Wu, R. Dunne, Z. Yu and W. Shi, "STREMS: A Smart Real-Time Solution toward Enhancing EMS Prehospital Quality," 2017 IEEE/ACM International Conference on Connected Health: Applications, Systems and Engineering Technologies (CHASE), Philadelphia, PA, 2017, pp. 365-372. https://doi.org/10.1109/CHASE.2017.120

[13] M. Poulymenopoulou, F. Malamateniou, and G. Vassilacopoulos, "Emergency healthcare process automation using mobile computing and cloud services," Journal of medical systems, vol. 36, no. 5, pp. 3233-3241, 2012. https://doi.org/10.1007/s10916-011-9814-y

[14] M. Toahchoodee, "ARSA-the pervasive Rescuer Supporting System for the Pre-hospital Emergency Medical Service," 2017 14th International Joint Conference on Computer Science and Software Engineering (JCSSE), NakhonSiThammarat, Thailand, 2017, pp. 1-6. https://doi.org/10.1109/JCSSE.2017.8025920

[15] E. Kyriacou, S. Christou, G. Hadjichristofi, R. Constantinou, A. Panayides and C. Pattichis, "Prehospital health care management platform," 2014 4th International Conference on Wireless Mobile Communication and Healthcare - Transforming Healthcare Through Innovations in Mobile and Wireless Technologies (MOBIHEALTH), Athens, 2014, pp. 152152. doi: 10.1109/MOBIHEALTH.2014.7015932.

[16] AZUMA, P. M. (2007). ISO/IEC 9126-4: Software Engineering - Software product quality - Part 4 : Quality in use metrics.

[17] Lewis, J. R. Psychometric evaluation of an after-scenario questionnaire for computer usability studies: The ASQ. SIGCHI Bulletin 23, 1 (1991), 78-81. https://doi.org/10.1145/122672.122692

\section{Authors}

Osman A. Abdellah, Majed M. Aborokbah, and Abdelrahman Osman Elfaki are with University of Tabuk, College of Computers and Information Technology, Tabuk, Saudi Arabia.

Article submitted 14 December 2017. Final acceptance 21 January 2018. Final version published as submitted by the authors. 